\title{
Using Quick Response Codes For Student Interaction During Lectures
}

\author{
Robert Law \\ Computer, Communications and Interactive Systems \\ Glasgow Caledonian University \\ Glasgow, Scotland \\ e-mail: robert.law@gcu.ac.uk
}

\begin{abstract}
This paper will present an ongoing project to encourage student interaction during lectures through the use of Quick Response (QR) codes and Google forms to generate rapid response polls and quizzes. The use of Google applications (Apps) software and the students' own mobile phone presents a free alternative to the current clicker systems. The pedagogical issues associated with such a project will be investigated and an attempt made to incorporate these into the student experience. The overall process from the creation of the software to the roll out and use of the software in an interactive lecture, the issues encountered and participant feedback will also be described.
\end{abstract}

Keywords- QR Codes; Student Interaction; Feedback.

\section{INTRODUCTION}

Smartphones in the UK, and elsewhere, have seen a surge in popularity over the last few years evidenced by recent figures showing "Over half of the British population (50.3\%) now owns a smartphone" [1]. Edinburgh University recently conducted a survey of their student population determining that $67 \%$ had ownership of a smartphone "an increase of seventeen percent from those students surveyed seven months earlier" [2]. This uptake in smartphone ownership within the student population opens a new dimension for interaction.

A tangible increase and use of Quick Response (QR) codes by many companies as a form of marketing has ensued on the back of this increase in smartphone popularity. This has allowed many companies to develop new and engaging ways for their customer base to interact with products in situ reinforcing brand presence. This potential has already been harnessed by Education to extend learning materials through the use of QR codes. Learning materials have been enhanced by providing "just in time support materials" [3] such as videos, explanatory text, Uniform Resource Indicators (URI) and staff details.

Using this as a platform to build from the next logical step is to combine the technologies to allow students to interact during lectures through quick multiple choice based questions. The students' responses can be compiled to show the result in a timely manner [4].

The remainder of this paper is organized as follows: Section II gives information about pedagogical issues related to interactive lectures, Section III introduces the technology used for implementing interactive lectures; this covers both hardware and software. Section IV discusses the authors experience of implementing interactive lectures, while Section V discusses issues encountered during the interactive lectures. Section VI offers a summary of our experience of interactive lectures, and concludes the paper giving proposals for future work.

\section{PEDAGOGICAL ISSUES}

Take a typical lecture; what does this encompass? Information is imparted upon the student in a relatively one way passive communication format. This traditional didactic approach is a format that has been used for centuries. This research intends to explore the possibility of improving and enhancing the lecture experience through the use of technology, and in particular, Audience Response Systems (ARS). The ability of such systems to encourage active learning through student participation and engagement provides an opportunity for enhancing the passive lecture format by introducing two way interactions with the student audience [7].

Murphy and Sharma further suggest that the research literature available for the topic of interactive lectures and the related pedagogical issues are "almost non-existent, with major issues waiting to be examined... inadequate research on the pedagogical implications of the emerging interactive forms of learning" [7]. With this in mind there appears to be an opportunity to examine and suggest how ARS technology could be used to not only enhance lecturer-student interactions, but also develop the underlying pedagogical issues inherent with lectures.

ARS technology provides a means for the lecturer to engage and interact with the students using the responses to offer the student audience feedback. This should lead to further discussion and the opportunity for student reflection. Other research, reported in Murphy and Sharma [7], identifies two pedagogical aspects of interactive lecturing: dialogic form of learning and active learning.

The project intends to examine these issues and the resultant effects that they have on the student audience. The primary concern is that the interactive lecture will stimulate engagement and interaction with the student audience and 
the lecturer through the use of instant feedback. This feedback will engender in both the student audience and the lecturer the need for reflection on many aspects of the material delivered and possibly the module in general. Through the use of relevant and targeted questions the students can be cajoled into discussions that will help expand their understanding of the topic area. Through these discussions both the students and the lecturer will be able to better understand the level of the students understanding of the subject area.

Gannon-Leary et al. [5] reported a number of other positive aspects to arise from interactive lectures including improved concentration, greater enjoyment and improved attendance. Simpson et al. [8] also noted that the anonymity provided by ARS technology played an important part in encouraging students to contribute to answering questions but suggests, as does Gannon-Leary et al. [5], that the design of the questions is very important to the process.

Saravani and Clayton [9] have developed a conceptual framework referred to as A.C.E. This framework is composed of the three A's: Awareness, Action, and Accomplishment; three C's: Context, Content, and Capability; and the three E's: Enabled, Engaged, and Empowered. The three E's aspect of the framework fits the concept driving interactive lectures as the use of mobile technology enables, engages and empowers both the student body and the lecturer.

Ramsden [3] suggests, amongst other things, that Quick Response (QR) codes can be used for "just in time information in a face to face lecture"; drawing on this point allows for the expansion of the concept to include feedback for both the student and the lecturer. Mooted by Law and So [11] is the idea that QR codes can facilitate a "trinity of "location independence," "time independence" and "meaningful content"'. Of interest in this "trinity" is the idea of "location independence"; being able to deliver and receive feedback to and from the students in the lecture hall.

\section{TECHNOLOGY}

ARS systems are available in many forms and price points. A typical classroom package can cost $\$ 1000$ or more for software, receiver and 12 clickers [12]. Some systems require the student to purchase a clicker and yearly registration at a cost of $\$ 20 / \$ 15$, respectively [12]. This project developed a "no cost in-house" system that was based on three key components: smartphones, QR codes and a Google spreadsheet. No proprietary software for the phone is required simply a standard browser and bar code reader. The "back-end" is relatively simple to create as the implementation interface is supplied by Google.

\section{A. Hardware}

The student participants referred to in Section IV were surveyed to determine the spread of handset manufacturers and phone operating systems. Around 50 students were surveyed.

Figure 1 shows the spread of handset manufacturers and Figure 2 shows the spread of phone operating systems with the surveyed population.
With such a range of manufacturers and operating systems an "app" based solution would be time consuming and prohibitive. With further investigation, it was discovered that third party barcode reading software was available for all the platforms, thus allowing the students to use their own phones for participation in the lectures.
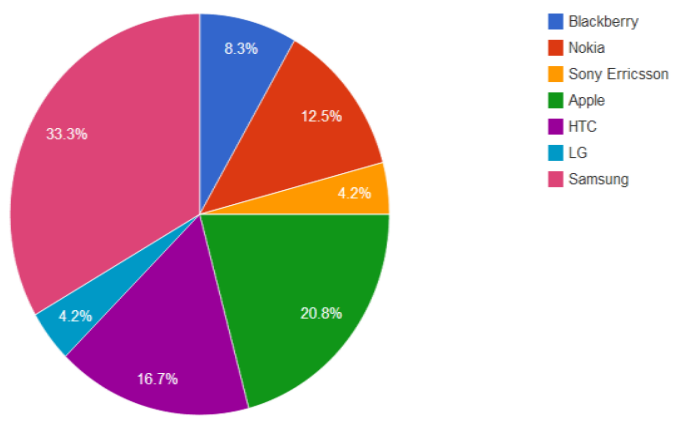

Figure 1. Spread of manufacturers
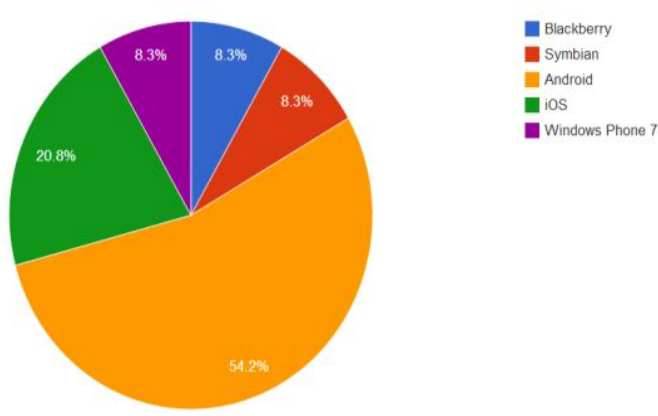

Figure 2. Spread of operating systems

\section{B. Software}

The software can be split into three categories: third party barcode reading software, third party browser software and the development of the interactive engine software using Google spreadsheets. It was up to the students themselves to decide on a suitable third party barcode reading software, although some phones did have such software preinstalled.

Three components of Google spreadsheets were used in the creation of the software application: the spreadsheet, the form and Google Script.

The spreadsheet itself is used as a repository for the student responses and also to house a summary sheet which keeps a running total of the number of responses for each possible answer to the question. The work horse of the system is the form and the scripts generated to process the responses at the back end. When a spreadsheet is created using Google Drive, a unique identifier is generated to identify the spreadsheet. When the subsequent form is created for the spreadsheet, another unique identifier is generated.

Although Google forms can handle a number of different inputs, the decision was taken to keep the question to a simple multiple choice style question, thus presenting the student with two or three QR codes per question. To create the $\mathrm{QR}$ codes requires the compilation of an http 
request based on the URI for the Google spreadsheet and the data to be sent to the spreadsheet. Once the http request was constructed and tested an online QR code generator was used to generate the required $\mathrm{QR}$ codes. These $\mathrm{QR}$ codes were subsequently saved as image files for insertion into the lecture slides.

Google script was used to create code that processed the student responses as they were received to generate a response summary that was visible to the student audience.

\section{Process}

The students used their smartphones to scan the QR code of their choice, submitting the data request via their phones browser. This, in turn, populated the spreadsheet with the students' choices, activating the script, allowing the results to be observed in near real time.

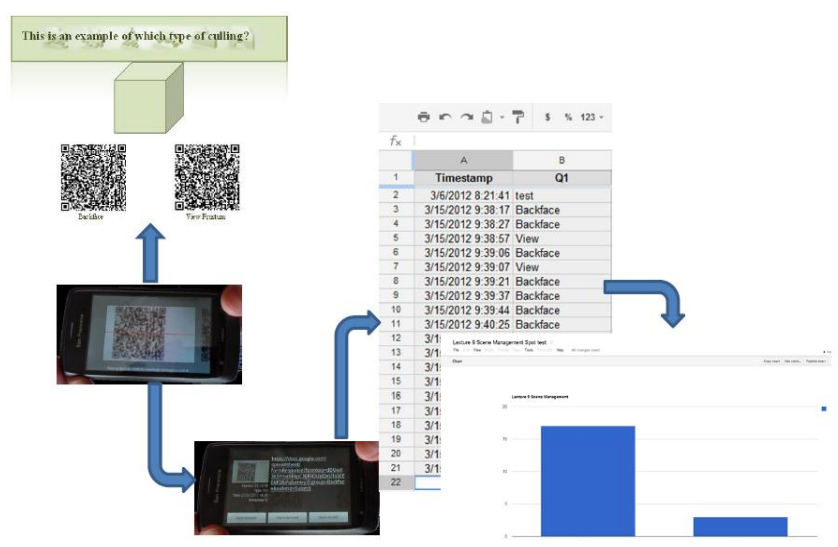

Figure 3. The interactive process

The QR Code is an encoded representation of the Google URI and data that will be sent to the spreadsheet when student scans it. As can be seen in Figure 3 above, once scanned, the information encoded in the $\mathrm{QR}$ code is decrypted and becomes visible to the phone's user. At this point, the participant has the ability to accept or decline the invite to send the data request. When the participant accepts the request to send the data, the next step is to invoke the phone's browser (this can work in different ways, depending on the phone/operating system) which will send the http request to Google for processing. Once processed, a "thank you" message is displayed in the browser indicating the data request has been received.

Once the data request has been received, the data is placed in the spreadsheet; the data will be based on the URI encoded in the QR Code. An example is shown below in Figure 4.

It is noticeable from Figure 4 that the spreadsheet date and time stamps the entries as it receives them. The only data that the spreadsheet records is the data encoded in the QR Code and the date and time stamp it generates on receipt of the data. Hence, all entries are anonymous.

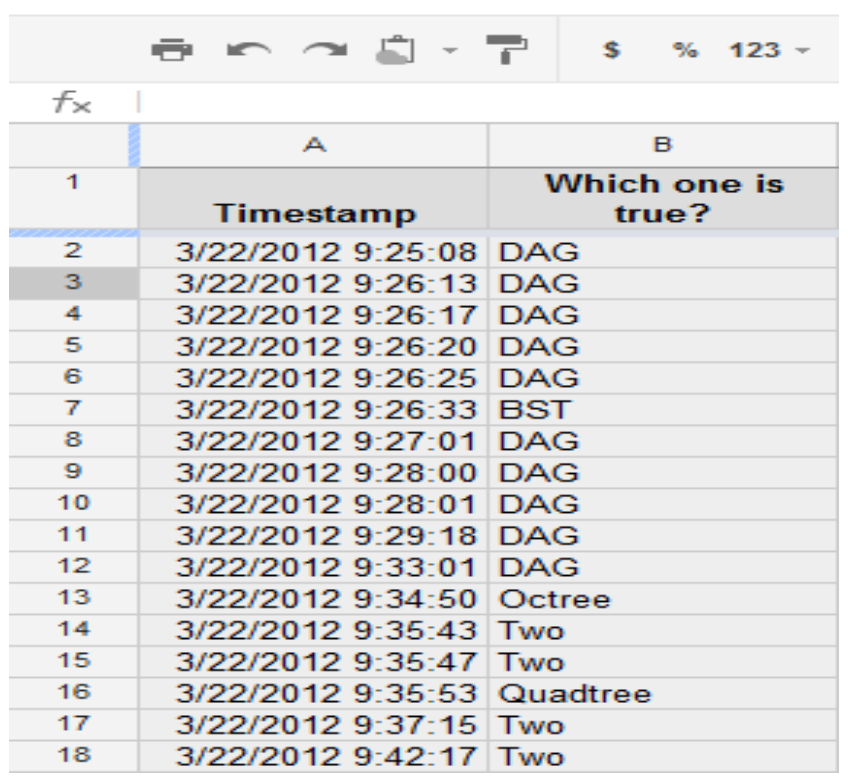

Figure 4. Data sent to the spreadsheet

\begin{tabular}{r|l|r|}
\hline$f_{X}$ & Rast & \\
\hline & & A \\
\hline 1 & Rast & 13 \\
\hline 2 & Geo & 6 \\
\hline 3 & Vertex & 0 \\
\hline 4 & Stencil & 12 \\
\hline
\end{tabular}

Figure 5. Summary sheet

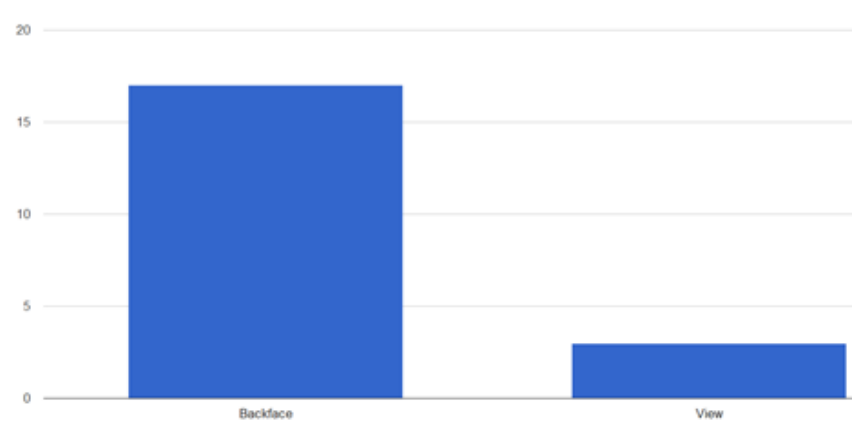

Figure 6. Generated bar chart

As each entry is received, a script is triggered, which counts the entries based on the predefined data set and populates a summary sheet which is used to generate the "near real time" bar charts.

An example of a summary sheet is shown in Figure 5 and an example of the bar chart displayed to the students is shown in Figure 6.

\section{EXPERIENCE}

A set of initial tests were developed to examine the viability of the technology and gauge the reaction of the 
students to the use of this technology within the lecture environment. The aim of the tests was to introduce the interaction concept in a gradual staged manner that wouldn't over burden the student or detract from the lecture.

\section{A. Test Setup}

The process used to create this interactive lecture was based on designing a set of suitable questions that could be used to strategically punctuate the lecture to gain maximum benefit for the students [13].

The desired effect was to integrate the technology within the lecture while stimulating interaction with the students [13]. As such, the first set of tests was built to increase in a systematic manner the number of questions within the lecture and the number of $\mathrm{QR}$ codes within the questions.

The structure of the first set of tests was designed to build from one question with one QR code in the lecture to four questions with three codes per question in the lecture. Suitable points within the lecture were identified such that the questions could be inserted to maximise their impact. An attempt was made to define suitable points through natural break points within the lecture e.g. end of a topic, end of the lecture, worked example. Using this principal, questions could be deployed with the aim of giving both the student and the lecturer instant feedback on the comprehension of the material delivered.

\section{B. Participants}

Both sets of participants were studying on the Games Software Development Degree. The first group to undertake the interactive lectures was a second year cohort of around 30 students and the second group to undertake the interactive lectures was a final year cohort of around 20 students.

The second year cohort had three consecutive lectures. The first of the three lectures had one question with two QR codes positioned at the end of the Lecture. The second Lecture had three questions, each with two QR codes positioned at appropriate points within the Lecture and the final Lecture had four questions, each with three QR codes again positioned at appropriate points within the Lecture.

The final year cohort had two lectures which were nonconsecutive. The first of the two lectures had two questions each with three $\mathrm{QR}$ codes positioned at appropriate points within the Lecture and the second Lecture had two questions each with three QR codes positioned at appropriate points within the Lecture.

At the appropriate point in the lecture, the slide would be displayed. To help minimize issues with scanning, paper copies of the slide were also distributed. This allowed for the difference within the quality of phone cameras to focus on the projected QR codes. It was fully explained to the students the nature of the experiment and the procedure which should be followed to correctly participate in the experiment.

\section{Feedback}

Initial feedback from both test groups has been positive and very informative. Feedback ranged from the ease of operation of the process to the size of the $\mathrm{QR}$ codes. In general a "buzz" was created within the participant groups generating a positive reaction from the students. This reaction must be tempered by the fact that the students are open to the "Hawthorne effect" [14] and, as such, more trials will need to be run to assess and define a clearer picture of the students reaction.

\section{ISSUES}

Although the overall outcome of the experiments was positive, a number of issues were highlighted that require further polishing prior to subsequent use.

Currently, the whole process required to create and integrate the QR codes into a lecture are quite cumbersome and may prove challenging for a non-computing subject specialist. An ongoing project has been put in place to integrate and automate the creation of the QR codes through a Web based Google spreadsheet application.

An issue flagged up by the participants centered on the size and positioning of the QR codes as this can have an impact on the accuracy of the scanning process.

The student participants indicated that the size of the QR codes on a projected slide proved difficult to scan directly. Not all students were able to scan it directly. This had been anticipated and paper based copies of the slides were issued to counter that problem. The issue appears to lie with the quality of the camera supplied with the phone.

Positioning of the QR Codes on the slide raised debate with the participants as some indicated that the barcode scanner software could find it difficult to focus on the required code. This seemed to be more prevalent when the codes were positioned side by side horizontally rather than stacked vertically. The number of QR codes on the slide also influenced the ease of scanning, with three codes per slide proving more challenging for the phone's barcode scanner. This was not insurmountable, but merely added a small time overhead as the participants positioned their camera phone to optimize the scan.

During one trial, a small number of participants encountered an issue with the barcode scanner software they were using. One student managed to automatically scan and send the same QR Code a number of times which did skew the graph for that particular question. On the lighter side, it did add a slice of humor, as the graph seemed to be growing by more than there were participants! On investigation, the student concerned indicated that the problem had arisen due to a setting in the third party barcode scanner software.

An intermittent issue, which arose on one occasion, was the apparent delay/failure of the chart to update itself as the data was received. This led to the chart being manually refreshed which did have an effect on the desired impact of the interactivity of the lecture.

Time management of both the interactions and the subsequent discussions should be built into the lecture timings allowing for leeway should anything go awry with the technology.

This approach relies on all the students in the lecture having a smartphone and it is conceivable that a small percentage of the student audience may in fact not have a smartphone or even a mobile phone. The perception would be that this student would be disadvantaged by not being 
able to take part in the interaction. This would be, of course, true. However, it could be mooted that the student is still engaged in the wider discussion that will come from viewing the generated graph. Another possibility is to rely on the goodwill of a fellow student to share access to their phone.

\section{CONCLUSION AND FUTURE WORK}

The overall trials were generally well received by the students serving the purpose of generating positive interaction between the lecturer and the students.

Students appeared to enjoy the break in the lecture and the feedback and discussion generated by the visual charting of their responses. It also created a focus point for the students to reflect on their understanding of the material taught and to apply that understanding. By the same token, it proved to be beneficial to the lecturer indicating the level of understanding of the delivered material to the students.

The anonymity of the whole process was cited by a number of students as positive. They felt comfortable with the fact that they could answer the questions freely, getting them wrong and not feeling awkward in front of their peers.

The project is ongoing and the positive feedback received from the students indicates that it is a worthwhile pursuit for both the lecturer and the students.

With regard to performance, this prototype system works well, producing the column chart of responses in near real time. More evaluation of the systems performance against other ARS systems is required. Initial use suggests promise with this cloud based system. Advantages this system offers is the fact it is free, flexible, easily tailored to suit the lecturer's needs and platform independent.

A further avenue for investigation will be the correct utilization and positioning of the interactive lectures within the overall module lecture delivery schedule. Over or under use will have an impact on their effectiveness.

Further investigation will be made with regard to the sizing, positioning and visibility of QR Codes from projected and paper based slides.

The use of the technique within the tutorial/seminar setting to encourage more debate on theoretical and social subjects is path that will be followed.

Further investigation will be undertaken into the relative pros and cons of storing complex responses in the spreadsheet, as evidenced in figure 4, and simplistic responses in the form $\mathrm{A}, \mathrm{B}, \mathrm{C}$, etc. The outcome of this investigation will have an impact on the future development of the software.

A significant proportion of future work will be involved in developing a user friendly interface to the software to allow cross discipline use. The script code will be hidden from the user allowing them to concentrate on the development of their question bank.

\section{REFERENCES}

[1] Kantar Worldpanel, The smartest way to communicate: over half of the GB population owns a smartphone. Available: http://goo.gl/Z1n7u. [retrieved: July, 2013].

[2] EUSA, Campus app now available for download. Available: http://www.eusa.ed.ac.uk/news/article/6001/290/. [retrieved: July , 2013].

[3] A. Ramsden, "The use of QR codes in Education: A getting started guide for academics.” Working Paper. University of Bath., 2008.

[4] R.Law, Using Quick Response Codes for Student Interaction During Lectures, ITiCSE, ACM 978-1-4503-1246, 2012.

[5] P. Gannon-Leary, C. Turnock, and M. McCarthy, "RECAP series paper 15", 2007.

[6] R.C. Lowery, "Teaching and learning with interactive student response systems: A comparison of commercial products in the higher-education market", 2005

[7] R. Murphy, and N. Sharma, "What Don't we know about interactive lectures?", Seminar. net-International journal of media, technology and lifelong learning, 2010, pp. 111.

[8] V. Simpson and M. Oliver, "Electronic voting systems for lectures then and now: A comparison of research and practice", Australasian journal of educational technology, vol. 23, no. 2, 2007, pp. 187.

[9] S.A Saravani and J.F. Clayton, A conceptual model for the educational deployment of $\mathrm{QR}$ codes. In Same places, different spaces. Proceedings ascilite Auckland 2009.

[10] V. Yfantis, P. Kalagiakos, C. Kouloumperi, and P. Karampelas, "Quick response codes in E-learning", Education and e-Learning Innovations (ICEELI), 2012 International Conference, 2012, pp. 1.

[11] C.Y. Law, and W.W.S. So, "QR codes in education", Journal of Educational Technology Development and Exchange, vol. 3, no. 1, 2010, pp. 85-100.

[12] E. Bojinova and J. Oigara, "Teaching and learning with clickers: Are clickers good for students", Interdisciplinary Journal of E-Learning and Learning Objects, vol. 7, 2011, pp. 169-183.

[13] A.P. Fagen, C.H. Crouch, and E. Mazur, "Peer instruction: Results from a range of classrooms", Physics Teacher, vol. 40, no. 4, 2002, pp. 206-209.

[14] S.R. Jones, "Was there a Hawthorne effect?", American Journal of Sociology, 1992, pp. 451-468. 